\title{
Avaliação do Risco de Parto Prematuro: Teste da Fibronectina Fetal e Medida do Colo Uterino
}

\author{
Evaluation of Risk for Preterm Delivery by Fetal Fibronectin \\ Test and Measurement of Uterine Cervix
}

\begin{abstract}
Tenilson Amaral Oliveira ${ }^{1,2}$, Carla Muniz Pinto de Carvalho ${ }^{1}$, Eduardo de Souza ${ }^{1}$, Jorge F. Kuhn dos Santos ${ }^{1,2}$
\end{abstract} Sandra de Oliveira Guaré ${ }^{1}$, Corintio Mariani-Neto ${ }^{2}$, Luiz Camano ${ }^{1}$

\section{RESUM0}

Objetivo: avaliar o risco de parto prematuro em gestantes com antecedentes de parto pré-termo espontâneo por meio do teste da fibronectina fetal e da medida do colo uterino pela ultrasonografia transvaginal.

Métodos: foram relacionadas 107 gestantes na 24a $28^{a}$ e $32^{\underline{a}}$ semana de gestação para realização do teste da fibronectina fetal no conteúdo cérvico- vaginal. No mesmo período, o comprimento do colo uterino foi medido, entre o orificio interno e externo, pela ultra-sonografia transvaginal. Consideramos o colo curto quando a medida da cérvice foi menor ou igual ao ponto de corte estabelecido pela curva ROC ("receiver-operating characteristic") para predição do parto prematuro. Comparamos o resultado dos exames com a ocorrência do parto antes de 34 e 37 semanas de gestação.

Resultados: a incidência do parto prematuro foi de 37,4\% (40/107). O melhor ponto de corte do comprimento do colo uterino indicado pela curva ROC para maximizar sensibilidade $e$ especificidade foi $30 \mathrm{~mm}$ para 24 e 28 semanas de gestação e $25 \mathrm{~mm}$ para 32 semanas. O teste positivo da fibronectina fetal teve um risco relativo (RR) significante apenas na $28^{a}$ semana (RR: 1,77; intervalo de confiança (IC) 95\%: 1,10-2,84) para a ocorrência do parto antes de 37 semanas. $O$ colo curto mostrou um $R R$ significativo para ocorrência do parto antes de 37 semanas, na $24^{a}, 28^{a}$ e $32^{a}$ semana. O RR foi mais elevado quando o colo curto esteve presente na $24^{a}$ semana para ocorrência do parto antes de 34 semanas (RR: 4,42; IC 95\%: 1,25-15,56).

Conclusão: em pacientes com antecedentes de prematuridade espontânea, a medida do comprimento do colo uterino por meio da ultra-sonografia transvaginal é melhor que o teste da fibronectina fetal para avaliar o risco de parto prematuro.

PALAVRAS-CHAVE: Fibronectina fetal. Colo uterino. Parto prematuro. Ultra-sonografia transvaginal.

\section{Introdução}

O parto prematuro é a principal causa de morbidade e mortalidade perinatal ${ }^{1,2}$. Apesar do melhor conhecimento dos fatores envolvidos na parturição e dos recursos terapêuticos à disposição do obstetra para bloquear o trabalho de

${ }^{1}$ Disciplina de Obstetrícia da Universidade Federal de São Paulo - Escola Paulista de Medicina - UNIFESP/EPM

${ }^{2}$ Hospital Maternidade "Leonor Mendes de Barros" Correspondência: Tenilson Amaral Oliveira

Rua Azevedo Júnior, $\mathrm{n}^{\circ} 143$ - apto. 73

03040-900 - São Paulo - SP

Fone: (11) 3399-5568 parto, a incidência de parto pré-termo não tem declinado nos últimos 50 anos ${ }^{3}$.

Deve ser enfatizado que não é conhecido o mecanismo pelo qual a parturição é iniciada, isto é, a preparação e o processo que leva à expulsão do produto conceptual, seja na gravidez a termo ou na pré-termo. Essa questão é fundamental para a seleção de estratégias ou tratamentos para a prevenção do parto prematuro.

A prematuridade pode acontecer espontaneamente, decorrente do trabalho de parto prematuro (TPP) propriamente dito, ou da interrupção da gravidez, devido a complicações mater- 
nas e fetais, que obrigam ou levam à inevitável ocorrência do parto antes de 37 semanas. Os trabalhos de Mann et al. ${ }^{4}$ e Arias e Tomich ${ }^{5}$ mostraram que cerca de 66 a $80 \%$ dos partos pré-termo são espontâneos. A prevenção da prematuridade eletiva exige a prevenção e/ou tratamento da pré-eclâmpsia, diabetes e outras doenças que levam à antecipação do parto ${ }^{6}$. A prevenção do parto prematuro espontâneo, por sua vez, é um dos principais desafios do obstetra na assistência pré-natal.

A incidência de prematuridade é cerca de três vezes maior se o primeiro parto foi prematuro, em relação às grávidas que tiveram o parto a termo ${ }^{7}$. Aproximadamente um terço das gestantes tem prematuridade em gravidez subseqüente a dois partos prematuros ${ }^{8}$.

Os fatores de risco não permitiram, até o momento, obter uma redução acentuada na incidência do parto pré-termo. Vários índices preditivos foram elaborados com esse objetivo, baseados na história pregressa e fatores de risco presentes durante a gravidez. Apesar de os trabalhos não terem sido prospectivos, os resultados, ao final, mostraram valores preditivos positivos (VPP) de aproximadamente 15 a 30\%, e sensibilidades que variaram de 35 a $60 \%{ }^{5}$.

A detecção da fibronectina fetal (FNF) nas secreções cérvico-vaginais no segundo e terceiro trimestres da gestação pode servir como um importante marcador bioquímico para o parto prematuro. Lockwood et al. ${ }^{9}$ e Bittar et al. ${ }^{10}$ identificaram as gestantes que tiveram parto prematuro por meio da pesquisa da FNF na secreção cérvico-vaginal em pacientes assintomáticas de alto risco. Tais resultados indicaram que a FNF tem valor clínico para a predição do parto prétermo.

A avaliação do colo uterino pela ultra-sonografia, por sua vez, permite detectar pequenas mudanças da cérvice uterina antes da manifestação clínica do TPP. Desse modo, pode servir como um importante elemento de conduta em pacientes com risco de prematuridade.

Embora a avaliação digital do colo uterino faça parte da rotina de pacientes que apresentam risco elevado de prematuridade, esse método não se mostra, na maioria das vezes, meio seguro de reconhecer alterações cervicais precoces. Yamasaki et al. ${ }^{11}$ verificaram que o comprimento do colo pela ultra-sonografia transvaginal (UST) apresentou melhor acurácia no diagnóstico do parto prematuro do que o toque vaginal em gestantes com risco elevado para o parto prematuro. Pode-se correlacionar a dilatação cervical e o esvaecimento com o intervalo até o parto, mas não há nível de esvaecimento ou di- latação capaz de auxiliar o clínico a selecionar pacientes para observação e tratamento. Aparentemente, isso decorre da natureza subjetiva do exame, como indicado pela ampla variação de comprimentos cervicais $(6-34 \mathrm{~mm})$, referida por Iams et al. ${ }^{12}$ como tendo $50 \%$ de esvaecimento.

A principal vantagem da medida da cérvice uterina por meio da ultra-sonografia transvaginal é avaliar a porção acima do fórnix anterior, o que não é possível com o toque vaginal. Da mesma maneira, o exame manual não consegue verificar a presença do funil endocervical. A medida do comprimento cervical por meio da ultrasonografia via abdominal apresenta limitações conforme a constituição física da paciente, posição da apresentação fetal e dificuldades técnicas, que podem tornar difícil a sua realização. Além disso, a necessidade do enchimento da bexiga não permite a padronização do método. O uso da UST tornou mais precisa a avaliação do comprimento do colo uterino, sendo superior ao exame digital como teste de predição do parto pré-termo ${ }^{13}$.

O objetivo do nosso trabalho foi comparar o valor clínico do teste da fibronectiona fetal e da medida do comprimento do colo uterino pela ultra-sonografia transvaginal na predição do parto prematuro espontâneo em pacientes com antecedentes de prematuridade.

\section{Pacientes e Métodos}

Realizou-se um estudo prospectivo em ambulatório pré-natal específico da Universidade Federal de São Paulo - Escola Paulista de Medicina (UNIFESP-EPM) e Hospital Maternidade "Leonor Mendes de Barros". Após aprovação pelas respectivas Comissões de Ética em Pesquisa, iniciamos nosso trabalho incluindo as gestantes que apresentassem em sua história obstétrica ao menos um parto prematuro espontâneo. As pacientes que apresentavam idade gestacional maior que 20 semanas e não tinham realizado ainda o exame de ultra-sonografia não foram selecionadas. Também foram excluídos do nosso estudo os seguintes casos: gestações com malformação fetal, insuficiência ístmica, gemelidade, inserção baixa de placenta, malformação uterina e prematuridade eletiva.

Após analisar os critérios de inclusão e exclusão e obter o consentimento das pacientes, realizamos o teste de imunoensaio de membrana na 24a․ 28a e $32^{a}$ semana. A presença da FNF no conteúdo cervical foi determinada qualitativamente utilizando um imunoensaio de mem- 
brana contendo o anticorpo monoclonal FDC-6, com ponto de corte para resultado positivo $\geq 50$ $\mathrm{ng} / \mathrm{ml}$ (Adeza Biomedical, Califórnia, EUA). Também medimos o comprimento do colo uterino pela UST (Figura 1) ao final das consultas prénatais no mesmo período. Para realização do exame, utilizamos equipamento com imagem de tempo real, Shimadzu, modelo SDU - 500C, munido de transdutor vaginal convexo de $5 \mathrm{MH}_{z}$ de freqüência.

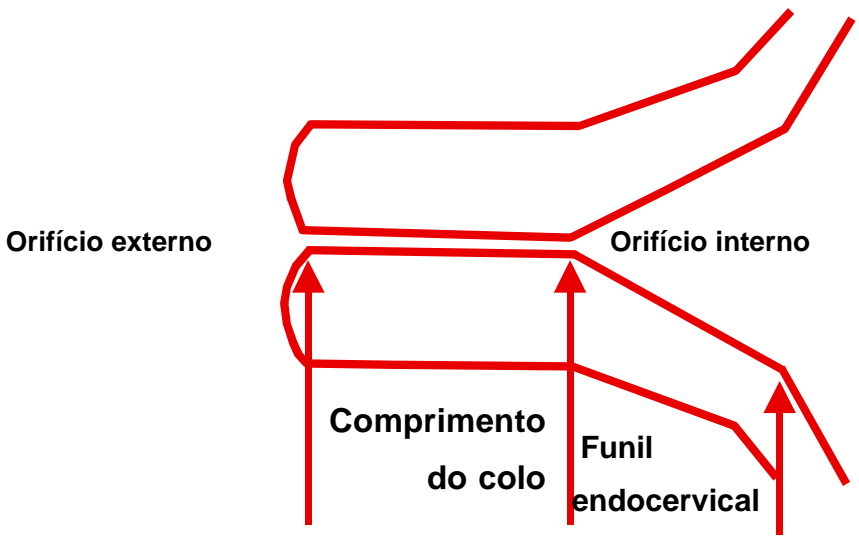

Figura 1-Medidas usadas para avaliação do colo uterino pela ultra-sonografia transvaginal.

Adotamos os seguintes critérios para assegurar uniformidade na medida do comprimento do colo uterino e reduzir a variabilidade intraobservador: o orifício interno do colo devia ser claramente visível como uma depressão suave, um triângulo isósceles ou em forma de funil; todo o comprimento do canal cervical deveria ser claramente visível; o orifício externo do colo visibilizado simetricamente; a superfície externa da cérvice claramente identificada; o funil endocervical, caso presente, não era incluído na medida do comprimento do colo.

O comprimento da cérvice uterina foi medido três vezes durante o mesmo exame e anotados seus resultados para posterior análise estatística. Todas as medidas do colo foram feitas sempre pelo mesmo examinador. Definimos o menor valor encontrado como sendo o comprimento do colo uterino.

Conforme as normas de conduta que utilizamos no pré-natal, não foi prescrito rotineiramente qualquer medicamento, de forma profilática, para evitar a ocorrência do TPP.

A medida do colo uterino pela UST e a coleta da FNF foram realizadas por dois examinadores diferentes. O resultados dos testes da FNF não foram mostrados ao ultra-sonografista e viceversa. O ultra-sonografista também não tinha conhecimento das medidas do colo feitas anteriormente, quando o exame foi realizado na $28^{\mathrm{a}} \mathrm{e}$
32 a semana de gestação. A equipe responsável pelo atendimento à paciente no pronto-socorro ou na enfermaria não teve acesso aos resultados da medida do colo ou do teste da FNF.

Consideramos parto prematuro, ou parto pré-termo, quando o nascimento do concepto ocorreu antes de 37 semanas completas de gestação. Analisamos também o resultados dos testes para a predição do parto antes de 34 semanas.

Para tentar estabelecer relações entre o resultado do parto (antes de 34 e 37 semanas) e o comprimento do colo foram estimadas curvas ROC e definidos os melhores pontos de corte. Denominamos o colo uterino como curto quando o comprimento da cérvice foi menor ou igual ao ponto de corte estabelecido pela curva ROC, de acordo com a idade gestacional.

A concordância entre a presença de alterações nos testes da FNF e no comprimento do colo uterino, relacionados à idade gestacional do parto, foi avaliada pelas medidas de sensibilidade, especificidade, VPP e valor preditivo negativo (VPN). A relação entre o resultado do teste da FNF e o comprimento do colo uterino foi analisada através do teste Kappa.

Foi calculado o risco relativo (RR) para cada parâmetro com 95\% de intervalo de confiança (IC), dispondo-se da posterior contraprova, isto é, a idade gestacional do parto.

Em todos os testes, foi fixado em 0,05, ou $5 \%$, o nível de significância.

\section{Resultados}

Participaram inicialmente do estudo 112 gestantes com antecedentes de parto prematuro. Duas grávidas não retornaram às consultas de pré-natal nas semanas subseqüentes à inclusão no estudo. Também foram excluídas da análise duas gestantes devido a óbito fetal ocorrido antes da $37^{\text {a }}$ semana e uma outra paciente por prematuridade eletiva (cardiotocografia alterada). Restaram, portanto, 107 gestantes para avaliação do risco de parto prematuro.

Entre as 107 gestantes, ocorreram 40 partos antes de 37 semanas $(37,4 \%)$ e 12 antes da $34^{\mathrm{a}}$ semana $(11,2 \%)$. Não verificamos óbito intrahospitalar de recém-nascidos.

Os melhores pontos de corte definidos pela curva ROC, de acordo com a idade gestacional para predição do parto prematuro, foram: $30 \mathrm{~mm}$ para 24 e 28 semanas e $25 \mathrm{~mm}$ para 32 semanas. Os pontos de corte foram os mesmos, em cada idade gestacional, não somente para pre- 
ver o parto antes de 37 semanas como também antes de 34 semanas. A Figura 2 mostra o ponto de corte obtido na $24^{\text {a }}$ semana segundo a curva ROC.

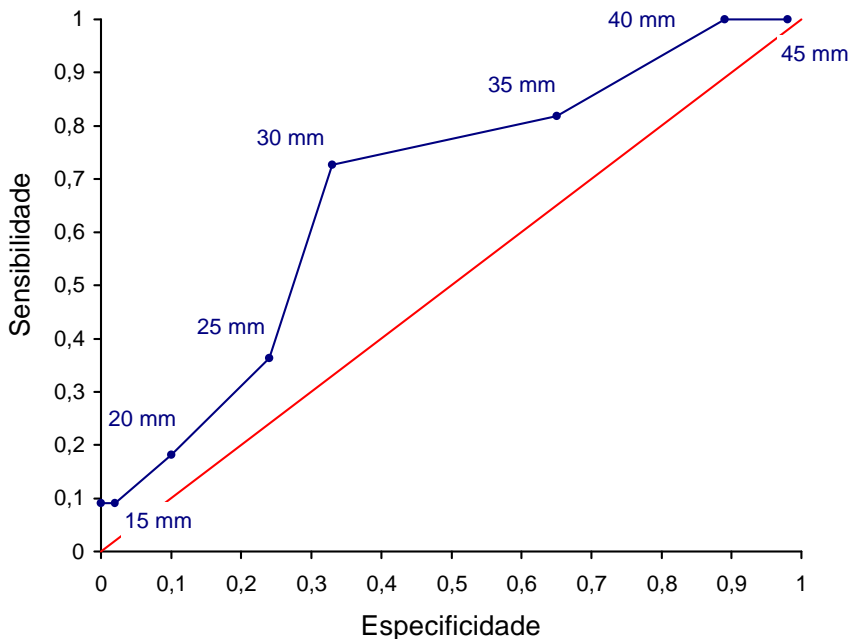

Figura 2 - Curva ROC para a previsão de parto prematuro antes de 34 semanas, utilizando a medida do comprimento do colo uterino pela ultra-sonografia transvaginal na 24a semana de gestação.
A Tabela 1 mostra os valores preditivos de acordo com o teste da FNF e a idade gestacional do parto antes da $34^{\text {a }}$ e $37^{\text {a }}$ semana, respectivamente. O RR foi significativo $(1,8$; IC $1,1-2,8)$ apenas na $28^{\mathrm{a}}$ semana para predição do parto antes de 37 semanas.

A Tabela 2 mostra os valores preditivos de acordo com o comprimento do colo uterino e a idade gestacional do parto antes de 34 e 37 semanas, respectivamente. Ocorreu RR significativo $(4,4$; IC $1,3-15,6)$ para predição do parto antes de 34 semanas, somente quando a medida da cérvice foi $\leq 30 \mathrm{~mm}$ na $24^{\text {a }}$ semana. Em relação a 37 semanas, o RR foi significante em todos os períodos analisados.

$\mathrm{O}$ resultado do teste FNF e o comprimento do colo tiveram seus resultados correlacionados significativamente com 24 e 28 semanas (Tabelas 3 e 4), o que não ocorreu na $32^{\text {a }}$ semana (Tabela 5).

Tabela 1- Sensibilidade, especificidade, valores preditivos positivo (VPP) e negativo (VPN) e risco relativo (RR) para o resultado do teste da fibronectina fetal na 24" $28^{\mathrm{a}}$ e $32^{\mathrm{a}}$ semana, com relação ao parto antes de 34 e 37 semanas.

\begin{tabular}{|c|c|c|c|c|c|c|c|c|c|c|}
\hline \multirow{3}{*}{$\begin{array}{l}\text { Teste da } \\
\text { fibronectina } \\
\text { fetal }\end{array}$} & \multirow{2}{*}{\multicolumn{2}{|c|}{$\begin{array}{c}\text { Sensibilidade } \\
\% \\
\text { Parto }\end{array}$}} & \multirow{2}{*}{\multicolumn{2}{|c|}{$\begin{array}{c}\text { Especificidade } \\
\% \\
\text { Parto }\end{array}$}} & \multirow{2}{*}{\multicolumn{2}{|c|}{$\begin{array}{l}\text { VPP } \\
\% \\
\text { Parto }\end{array}$}} & \multicolumn{2}{|c|}{$\begin{array}{l}\text { VPN } \\
\%\end{array}$} & \multicolumn{2}{|c|}{$\begin{array}{c}\text { RR } \\
\text { (IC 95\%) }\end{array}$} \\
\hline & & & & & & & $\begin{array}{r}9 \\
\mathrm{~Pa}\end{array}$ & & & \\
\hline & $<34$ sem & $<37 \mathrm{sem}$ & $<34 \mathrm{sem}$ & $<37 \mathrm{sem}$ & $<34$ sem & $<37 \mathrm{sem}$ & $<34$ sem & $<37$ sem & $<34 \mathrm{sem}$ & $<37$ sem \\
\hline $24 \mathrm{sem}$ & 22 & 39 & 67 & 71 & 7 & 41 & 89 & 69 & $\begin{array}{c}0,6 \\
(0,1-2,7)\end{array}$ & $\begin{array}{c}1,3 \\
(0,8-2,4)\end{array}$ \\
\hline $28 \mathrm{sem}$ & 42 & 44 & 71 & 78 & 16 & 55 & 90 & 69 & $\begin{array}{c}1,6 \\
(0,6-4,8)\end{array}$ & $\begin{array}{c}1,8 \text { * } \\
(1,1-2,8)\end{array}$ \\
\hline $32 \mathrm{sem}$ & 60 & 50 & 63 & 67 & 8 & 43 & 97 & 73 & $\begin{array}{c}2,4 \\
(0,4-3,7)\end{array}$ & $\begin{array}{c}1,6 \\
(0,9-2,8)\end{array}$ \\
\hline
\end{tabular}

${ }^{*} p<0,05$

Tabela 2 - Sensibilidade, especificidade, valores preditivos positivo (VPP) e negativo (VPN) e risco relativo (RR) para o comprimento do colo uterino $\leq 30 \mathrm{~mm}$ e $>30 \mathrm{~mm}$ na idade gestacional (IG) de 24 e 28 semanas e $\leq 25 \mathrm{~mm}$ e $>25 \mathrm{~mm}$ na IG de 32 semanas, com relação ao parto antes de 34 e 37 semanas.

\begin{tabular}{|c|c|c|c|c|c|c|c|c|c|c|}
\hline \multirow[t]{3}{*}{ Colo uterino } & \multirow{2}{*}{\multicolumn{2}{|c|}{$\begin{array}{c}\text { Sensibilidade } \\
\% \\
\text { Parto }\end{array}$}} & \multirow{2}{*}{\multicolumn{2}{|c|}{$\begin{array}{c}\text { Especificidade } \\
\% \\
\text { Parto }\end{array}$}} & \multicolumn{2}{|c|}{$\begin{array}{l}\text { VPP } \\
\% \\
\text { Parto }\end{array}$} & \multicolumn{2}{|c|}{$\begin{array}{c}\text { VPN } \\
\%\end{array}$} & \multicolumn{2}{|c|}{$\begin{array}{c}\text { RR } \\
\text { (IC 95\%) }\end{array}$} \\
\hline & & & & & & & & & $\mathrm{Pa}$ & \\
\hline & $<34$ sem & $<37$ sem & $<34$ sem & $<37$ sem & $<34$ sem & $<37$ sem & $<34$ sem & $<37$ sem & $<34$ sem & $<37$ sem \\
\hline 24 sem & 73 & 59 & 67 & 75 & 23 & 57 & 95 & 76 & $\begin{array}{c}4,4 \text { * } \\
(1,3-5,6)\end{array}$ & $\begin{array}{c}2,4 \text { * } \\
(1,4-4,1)\end{array}$ \\
\hline $28 \mathrm{sem}$ & 75 & 73 & 52 & 63 & 17 & 55 & 94 & 78 & $\begin{array}{c}2,9 \\
(0,8-10,1)\end{array}$ & $\begin{array}{c}2,6^{*} \\
(1,4-4,5)\end{array}$ \\
\hline 32 sem & 67 & 73 & 50 & 60 & 8 & 48 & 96 & 81 & $\begin{array}{c}1,9 \\
(0,4-0,0)\end{array}$ & $\begin{array}{c}2,6^{\star} \\
(1,3-4,9)\end{array}$ \\
\hline
\end{tabular}

${ }^{*} \mathrm{p}<0,05$ 
Tabela 3 - Relação entre o comprimento do colo uterino pela ultra-sonografia transvaginal e resultado do teste da fibronectina fetal na $24^{a}$ semana de gestação.

\begin{tabular}{lccc}
\hline Comprimento do colo & \multicolumn{2}{c}{ Teste da fibronectina fetal } \\
Positivo & Negativo & Total \\
\hline$\leq 30$ & 15 & 18 & 33 \\
$>30$ & 14 & 43 & 57 \\
Total & 29 & 61 & 90 \\
\hline
\end{tabular}

${ }^{*} p=0,041$

Tabela 4 - Relação entre o comprimento do colo uterino pela ultra-sonografia transvaginal e 0 resultado do teste da fibronectina fetal na $28^{a}$ semana de gestação.

\begin{tabular}{lccr}
\hline Comprimento do colo & \multicolumn{2}{c}{ Teste da fibronectina fetal } \\
Positivo & Negativo & Total \\
\hline$\leq 30$ & 24 & 29 & 53 \\
$>30$ & 7 & 42 & 49 \\
Total & 31 & 71 & 102 \\
\hline
\end{tabular}

${ }^{*} p=0,001$

Tabela 5 - Relação entre o comprimento do colo uterino pela ultra-sonografia transvaginal e 0 resultado do teste da fibronectina fetal na 32 a semana de gestação.

\begin{tabular}{lccc}
\hline Comprimento do colo & \multicolumn{2}{c}{ Teste da fibronectina fetal } \\
Positivo & Negativo & Total \\
\hline$\leq 25$ & 21 & 27 & 48 \\
$>25$ & 16 & 32 & 48 \\
Total & 37 & 59 & 96 \\
\hline
\end{tabular}

${ }^{*} p=0,294$

\section{Discussão}

Não há uma maneira precisa de estabelecer o momento em que é iniciada a parturição. Contudo, o início do processo envolve uma seqüência de modificações da função uterina, que levam o útero a se contrair de modo coordenado, forçando a dilatação e descida do feto pelo canal do parto. Quaisquer que sejam os fatores responsáveis pelo desencadeamento do TPP, a identificação precoce dessas gestantes possibilita a adoção de condutas, que podem ser agrupadas em duas categorias: as que buscam evitar a ocorrência do TPP e as que procuram inibilo. O sucesso de ambas as estratégias dependerá da qualidade dos instrumentos de predição e do nosso conhecimento dos mecanismos patológicos envolvidos nesse processo. Por isso, são necessários métodos preditivos eficazes, que possam ser utilizados principalmente para prever a ocorrência do parto antes de 34 semanas, tendo em vista que esse é o período de maior morbidade e mortalidade perinatal.
A incidência elevada do parto antes da $34^{\text {a }}$ e da 37ํㅗ semana em nossa casuística confirma o antecedente de que o parto prematuro espontâneo é um dos maiores fatores de risco para a ocorrência de subseqüente prematuridade.

$O$ teste da FNF mostrou índices baixos de sensibilidade e VPP para predição do parto prematuro não somente antes da $34^{\mathrm{a}}$ semana, como também antes da $37^{a}$ semana. O VPN, por outro lado, foi alto para predição da prematuridade antes da $34^{\mathrm{a}}$ semana nos três períodos analisados, apresentando, porém, riscos relativos não significativos. Iams et al. ${ }^{13}$ consideram que sensibilidade e VPN altos devem ser características essenciais para qualquer teste de triagem diante de uma intercorrência grave como a prematuridade.

Apesar dos baixos valores de sensibilidade, o teste exibiu na $28^{a}$ semana um RR significativo para predição do parto antes de 37 semanas. Nageotte et al. ${ }^{14}$ apuraram especificidade e VPP baixos em grávidas de alto risco. Peaceman et al. ${ }^{15}$ também verificaram VPP baixo nesse grupo de risco e VPN alto, sugerindo que menos intervenção possa ser a conduta mais apropriada para essas pacientes.

Os valores preditivos positivos baixos para o teste da FNF relacionado ao parto antes da $34^{-}$ e 37ํㅗ semana encontrados em nosso trabalho são semelhantes àqueles vistos na literatura ${ }^{9,13,14,16}$, independente do período do parto pré-termo considerado, número de amostras realizadas e grupo de risco.

Nossos resultados parecem indicar que o teste da FNF tem pouco valor em pacientes assintomáticas de alto risco, característica das pacientes com antecedentes de prematuridade.

Embora alguns autores tenham referido bons resultados para predição do parto pré-termo com a FNF, Goldenberg et al. ${ }^{16}$ concluíram que o uso da FNF não resultaria na redução da incidência de prematuridade e não teria maior impacto sobre a mortalidade perinatal, tendo em vista que na maioria dos trabalhos publica$\operatorname{dos}^{9,17,18}$, o peso médio dos recém-nascidos com teste positivo ultrapassou 2.200 gramas. Em recém-nascidos com peso superior a 2.000 gramas, medidas profiláticas e bloqueio do trabalho de parto não mostram, segundo os autores, benefícios perinatais significativos.

Os melhores pontos de corte para predição do parto prematuro por meio do comprimento cervical mostraram dois valores distintos na curva ROC: $30 \mathrm{~mm}$ e $25 \mathrm{~mm}$, o primeiro relacionado à $24^{\mathrm{a}}$ e à $28^{\mathrm{a}}$ semana de gestação e o último à $32^{\mathrm{a}}$ semana. Os resultados foram semelhantes aos encontrados nos trabalhos de Andersen et al. ${ }^{19}$, 
Murakawa et al. ${ }^{20}$ e Hasegawa et al. ${ }^{21}$, isto é, o comprimento do colo diminuiu à medida que a gestação avançou até o termo, reduzindo os limites considerados de alto risco.

Apesar de a medida do colo pela UST ter sido um marcador de risco significativo para o parto antes da $37^{\text {a }}$ semana em todos os períodos analisados, seu valor clínico, provavelmente, se deve ao elevado $\mathrm{RR}$ relacionado ao parto antes da $34^{\mathrm{a}}$ semana, quando o exame foi realizado na $24^{\text {a }}$ semana. Salientamos a importância de um parâmetro confiável para predição do parto antes da $34^{\text {a }}$ semana, devido à elevada morbidade e mortalidade nesse período.

A medida do colo uterino apresentou bons indices de VPN, principalmente para predição do parto antes da $34^{\mathrm{a}}$ semana. A especificidade e VPP, todavia, foram mais baixos. A sensibilidade observada em nosso estudo, na $24^{\text {a }}$ semana, para predição do parto antes de 34 semanas, foi superior ao encontrado por Iams et al. ${ }^{22}(54 \%)$ e semelhante ao valor verificado pelos mesmos autores na $28^{\mathrm{a}}$ semana $(76 \%)$.

O comprimento cervical mostrou melhor associação com o parto pré-termo do que a FNF. Além de relacionada ao parto antes da $34^{\mathrm{a}}$ semana, a presença de um colo curto também foi associada significativamente ao parto antes da $37^{\text {a }}$ semana em todos os períodos analisados. Por outro lado, o teste da FNF na $28^{\text {a }}$ semana foi mais específico, ao passo que o colo curto foi mais sensível, no mesmo período, para previsão do parto antes de 37 semanas.

A competência do colo uterino, avaliada como uma variável presente ou ausente, pode ocultar o significado de uma possivel incompetência cervical relativa. Iams et al. ${ }^{23}$ concluíram que a competência cervical é uma variável contínua, ao observarem que o tamanho do colo na gravidez subseqüente apresenta uma relação linear com a duração da gestação anterior. Essa incompetência relativa, tratada como uma variável contínua, permitiria quantificar a competência cervical como uma medida da resistência ístmica, que teria ou não a capacidade de manter a gravidez até o termo. Essa medida poderia corresponder indiretamente ao comprimento do colo uterino. A capacidade da cérvice seria refletida funcionalmente pela duração da gravidez e anatomicamente pelo seu comprimento ${ }^{23}$. Nossos dados não permitem conclusões sobre a incompetência relativa do colo uterino como fator causal do parto pré-termo, mas não podemos subestimar a importância desse fator, atribuindo a associação entre colo curto e parto pré-termo unicamente ao aumento da contractilidade uterina.
Atualmente, a conduta em gestantes com antecedentes de prematuridade está baseada nos agentes tocolíticos, que apresentam limitada eficácia. Talvez a melhor abordagem seja a avaliação do colo uterino. A presença de colo curto pode sugerir a necessidade de aumentar o seu comprimento. Heath et al. ${ }^{24}$ utilizaram a UST na população de baixo risco para rastreamento de pacientes com colo curto ( $\leq 15 \mathrm{~mm})$ na $23^{\text {a }}$ semana e posterior circlagem, obtendo melhores resultados perinatais no grupo que realizou o procedimento.

Como a ultra-sonografia é rotineiramente solicitada no pré-natal atual, a medida do comprimento do colo deveria ser item obrigatório do exame realizado em pacientes com antecedentes de parto prematuro.

Nossos dados sugerem que a presença do colo curto e do teste positivo da FNF tem potencial para servir como instrumentos de rastreamento para detecção do parto prematuro em gestantes com história de prematuridade. Os testes da FNF e a medida do colo, além disso, podem auxiliar o pré-natalista na orientação de medidas preventivas ou terapêuticas, sobretudo devido ao elevado VPN para ocorrência do parto antes de 34 semanas. A identificação precoce dessas pacientes serviria, por exemplo, para orientar a administração dos corticosteróides para acelerar a maturação pulmonar fetal.

\section{SUMMARY}

Purpose: to evaluate the risk of preterm delivery using the fetal fibronectin test and the measurement of the cervix by transvaginal ultrasonography in pregnant women with previous preterm delivery.

Methods: one hundred and seven women were enrolled in the study at 24th, 28th and 32nd week to detect the presence of fetal fibronectin by immediate-reading membrane test and to perform vaginal ultrasonography to measure the length of the cervix between the internal and external cervical os. The cervix was considered to be short when the cervical length was at or below the cutoff set by the receiveroperating characteristic (ROC) curve for prediction of preterm delivery. Sonographic cervical length and fetal fibronectin were compared to assess the risk of preterm birth before 34 and 37 weeks.

Results: the spontaneous preterm delivery rate was $37.4 \%$ (40/107). The analysis made by the ROC curve indicated $30 \mathrm{~mm}$ as the best cutoff to maximize sensitivity and specificity at 24 and 28 weeks and $25 \mathrm{~mm}$ at 32 weeks of gestation. The positive test of fetal fibronectin had a significant relative risk (RR: 1.77; 95\% confidence interval (IC): 1.10-2.84) to predict delivery before 37 weeks, when compared with a negative test, only at 28 weeks. The 
presence of short cervix at 24, 28 and 32 weeks showed a significative RR for birth before 37 weeks. The highest RR occurred with a short cervix at 24 weeks (RR: 4.42; 95\% CI: $1.25-15.56)$.

Conclusion: we concluded that the measurement of uterine cervix by vaginal ultrasonography is better than the fetal fibronectin test for evaluating the risk of preterm delivery in women with previous preterm delivery.

KEY WORDS: Fetal fibronectin. Uterine cervix. Preterm labor. Transvaginal ultrasonography.

\section{Referências}

1. Creasy RK, Merkatz IR. Prevention of preterm birth: clinical opinion. Obstet Gynecol 1990; 76 Suppl:2S-4S.

2. Morrison JC. Preterm birth: a puzzle worth solving. Obstet Gynecol 1990; 76 Suppl:5S-12S.

3. Craigo SD. Cervical incompetence and preterm delivery. N Engl J Med 1996; 334:595-6.

4. Mann LI, Baker DA, Gallant J. Dealing with the sources of low birthweight. Contemp Ob/Gyn $1981 ; 18: 141-5$.

5. Arias F, Tomich P. Etiology and outcome of low birth weight and preterm infants. Obstet Gynecol 1982; 60:277-81.

6. Iams JD, Johnson FF, Creasy RK. Prevention of preterm birth. Clin Obstet Gynecol 1988; 31:599615.

7. Carr-Hill RA, Hall MH. The repetition of spontaneous preterm labour. Br J Obstet Gynaecol 1985; 92:921-8.

8. Kristensen J, Langhoff-Roos J, Kristensen FB. Implications of idiopathic preterm delivery for previous and subsequent pregnancies. Obstet Gynecol 1995; 86:800-4.

9. Lockwood CJ, Senyei AE, Dische MR, et al. Fetal fibronectin in cervical and vaginal secretions as a predictor of preterm delivery. N Engl J Med 1991; 325:669-74.

10.Bittar RE, Yamasaki AA, Sasaki S, Zugaib M. Cervical fetal fibronectin in patients at increased risk for preterm delivery. Am J Obstet Gynecol 1996; 175:178-81.

11.Yamasaki AA, Bittar RE, Fonseca ESB, Martinelli S, Sasaki S, Zugaib M. Prevenção do parto prematuro: emprego do toque vaginal e da ultrasonografia transvaginal. Rev Bras Ginecol Obstet 1998; 20:350-6.

12.Iams JD, Paraskos J, Landon MB, Teteris JN, Johnson FF. Cervical sonography in preterm labor. Obstet Gynecol 1994; 84:40-6.

13.Iams JD, Casal D, McGregor JA, et al. Fetal fibronectin improves the accuracy of diagnosis of preterm labor. Am J Obstet Gynecol 1995; 173:141-5.

14.Nageotte MP, Casal D, Senyei AE. Fetal fibronectin in patients at increased risk for premature birth. Am J Obstet Gynecol 1994;170:20-5.

15.Peaceman AM, Andrews WW, Thorp JM, et al. Fetal fibronectin as a predictor of preterm birth in patients with symptoms: a multicenter trial. Am J Obstet Gynecol 1997; 177:13-8.

16.Goldenberg RL, Thom E, Moawad AH, Johnson F, Roberts J, Caritis SN. The preterm prediction study: fetal fibronectin, bacterial vaginosis, and peripartum infection. NICHD Maternal Fetal Medicine Units Network. Obstet Gynecol 1996; 87:656-60.

17.Lockwood CJ, Wein R, Lapinsk R, et al. The presence of cervical and vaginal fetal fibronectin predicts preterm delivery in an inner city obstetric population. Am J Obstet Gynecol 1993; 169:798804.

18. Morrison JC, Allbert JR, McLaughlin BN, Whitworth NS, Roberts WE, Martin RW. Oncofetal fibronectin in patients with false labor as a predictor of preterm delivery. Am J Obstet Gynecol 1993; 168:538-42.

19.Andersen HF, Nugent CE, Wanty SD, Hayashi RH. Prediction of risk for preterm delivery by ultrasonographic measurement of cervical length. Am J Obstet Gynecol 1990; 163:859-67.

20.Murakawa H, Utumi T, Hasegawa I, Tanaka K, Fuzimori R. Evaluation of threatened preterm delivery by transvaginal ultrasonographic measurement of cervical length. Obstet Gynecol 1993; 82:829-32.

21.Hasegawa I, Tanaka K, Takahashi K, et al. Transvaginal ultrasonographic cervical assessment for the prediction of preterm delivery. J Matern Fetal Med 1996; 5:305-9.

22.Iams JD, Goldenberg RL, Meis PJ, et al. The length of the cervix and the risk of spontaneous premature delivery. National Institute of Child Health and Human Development Maternal Fetal Medicine Unit Network. N Engl J Med 1996; 334:567-72.

23.Iams JD, Johnson FF, Sonek J, Gebauer C, Samuels P. Cervical competence as a continuum: a study of ultrasonographic cervical length and obstetric performance. Am J Obstet Gynecol 1995; 172:1097-106.

24.Heath VC, Souka AP, Erasmus I, Gibb DM, Nicolaides $\mathrm{KH}$. Cervical length at 23 weeks of gestation: the value of Shirodkar suture for the short cervix. Ultrasound Obstet Gynecol 1998; 12:31822 . 